Annales Geophysicae (2001) 19: 1567-1578 C) European Geophysical Society 2001

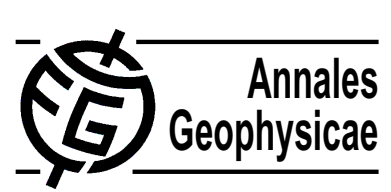

\title{
Four point measurements of electrons using PEACE in the high-altitude cusp
}

\author{
M. G. G. T. Taylor ${ }^{1}$, A. Fazakerley ${ }^{1}$, I. C. Krauklis ${ }^{1}$, C. J. Owen ${ }^{1}$, P. Travnicek ${ }^{1,2}$, M. Dunlop ${ }^{3}$, P. Carter ${ }^{1}$, \\ A. J. Coates ${ }^{1}$, S. Szita ${ }^{1}$, G. Watson ${ }^{1}$, and R. J. Wilson ${ }^{1}$ \\ ${ }^{1}$ Mullard Space Science Laboratory, University College London, UK \\ ${ }^{2}$ Institute of Atmospheric Physics, The Academy of Sciences of the Czech Republic \\ ${ }^{3}$ Imperial College of Science Technology and Medicine, UK
}

Received: 1 May 2001 - Revised: 2 July 2001 - Accepted: 11 July 2001

\begin{abstract}
We present examples of electron measurements from the PEACE instruments on the Cluster spacecraft in the high-latitude, high-altitude region of the Earth's magnetosphere. Using electron density and energy spectra measurements, we examine two cases where the orbit of the Cluster tetrahedron is outbound over the northern hemisphere, in the afternoon sector approaching the magnetopause. Data from the magnetometer is also used to pinpoint the position of the spacecraft with respect to magnetospheric boundaries. This preliminary work specifically highlights the benefit of the multipoint measurement capability of the Cluster mission. In the first case, we observe a small-scale spatial structure within the magnetopause boundary layer. The Cluster spacecraft initially straddle a boundary, characterised by a discontinuous change in the plasma population, with a pair of spacecraft on either side. This is followed by a complete crossing of the boundary by all four spacecraft. In the second case, Cluster encounters an isolated region of higher energy electrons within the cusp. The characteristics of this region are consistent with a trapped boundary layer plasma sheet population on closed magnetospheric field lines. However, a boundary motion study indicates that this region convects past Cluster, a characteristic more consistent with open field lines. An interpretation of this event in terms of the motion of the cusp boundary region is presented.
\end{abstract}

Key words. Magnetospheric physics (magnetopause, cusp and boundary layers; solar wind-magnetosphere interactions)

\section{Introduction}

The high-altitude, high latitude region of the magnetosphere has had sparse coverage by satellite observations. The POLAR satellite only reaches altitudes of around $9 R_{E}$ (Russell, 2000). Recently, the Interball missions (Zelenyi et al., 1997)

Correspondence to: M. G. G. T. Taylor

(mggtt@mssl.ucl.ac.uk) have flown in the cusp, with INTERBALL-TAIL reaching cusp altitudes of $\sim 10-11 R_{E}$. The INTERBALL-TAIL satellite was accompanied by a sub-satellite, MAGION-4, providing simultaneous observations of the high- and midaltitude cusp. Such observations have shown the cusp and surrounding boundary region to be well-defined (Sandahl et al., 2000) and to occupy a broader region (in latitude and longitude) than expected from low latitude observations (Merka et al., 2000). Prior to this, the last missions to this region were HEOS 1 and 2 (Hedgecock and Thomas, 1975) and Hawkeye (Farrell and Van Allen, 1990). The large (orbit) apogees of these spacecraft (HEOS at $\sim 37 R_{E}$ and Hawkeye at $21 R_{E}$ ) were such that they were able to take data from regions beyond the magnetopause boundary, allowing for coverage of the high-latitude, high-altitude boundary layers in and around the cusp. The cusp crossings from these satellites provided a greater understanding of the dynamics and geometry of the region, as described in Haerendel et al. (1978), Farrell and Van Allen (1990) and more recently in Kessel et al. (1996), Chen et al. (1997), Dunlop et al. (2000) and Eastman et al. (2000). Both HEOS and Hawkeye had rather low resolution plasma detectors. The HEOS full energy spectrum $(100 \mathrm{eV}-40 \mathrm{keV})$ was taken every $256 \mathrm{~s}$ and Hawkeye, every $210 \mathrm{~s}$. The INTERBALL-TAIL ELECTRON instrument provided 2 min resolution (Zelenyi et al., 1997) over an $10 \mathrm{eV}$ to $22 \mathrm{keV}$ range. In comparison, the Plasma Electron and Current Experiment (PEACE) instrument on board Cluster provides much higher time and energy resolution than its predecessors ( $\sim 0.6 \mathrm{eV}$ to $27 \mathrm{keV}$ with $4 \mathrm{~s}$ resolution), enabling an examination of much smaller-scale plasma phenomena.

This paper introduces two preliminary studies of four point measurements from the ESA Cluster mission. We have selected periods from the first 2 months of nominal operation to demonstrate the capability of Cluster in inferring the direction and structural configuration of magnetospheric features. In both cases, the Cluster spacecraft were travelling towards the magnetopause in the northern afternoon sector, nominally on the dusk side of the cusp, as shown in Fig. 1. The spacecraft were flying in a tetrahedron configuration with an aver- 

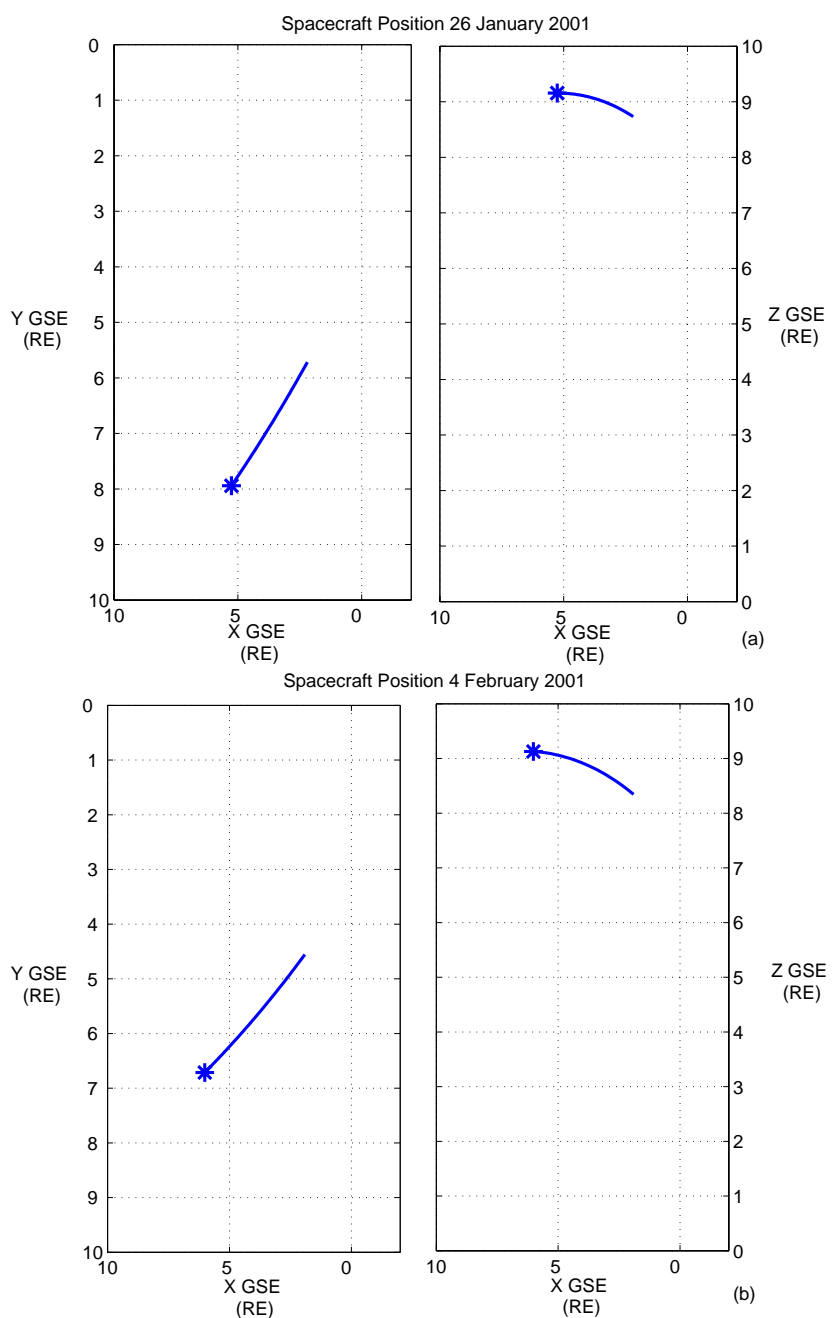

Fig. 1. The orbit position of the Cluster quartet for the two cases presented in this paper. The plots are in GSE coordinates with the Sun to the left. The star represents the end of the time period.

age separation of $\sim 600 \mathrm{~km}$ and at an altitude of $\sim 9-12 R_{E}$. In the next section, we briefly introduce the PEACE instruments. In Sect. 3, we describe the 2 cases, in turn, followed by a discussion of each event in Sect. 4, which begins with a brief overview of the boundary analysis technique used in this paper. Section 5 summarises the findings and discusses further work.

\section{The PEACE instrument}

The Plasma Electron and Current Experiment (PEACE) on board the Cluster spacecraft consists of two sensors, HEEA (High Energy Electron Analyser) and LEEA (Low Energy Electron Analyser), mounted on diametrically opposite sides of the spacecraft. They are designed to measure the three dimensional velocity distributions of electrons in the range of $0.6 \mathrm{eV}$ to $\sim 26 \mathrm{keV}$. In standard mode, HEEA measures the range of $35 \mathrm{eV}$ to $26 \mathrm{keV}$ and LEEA measures the range of $0.6 \mathrm{eV}$ to $1 \mathrm{keV}$, although either can be set to cover any

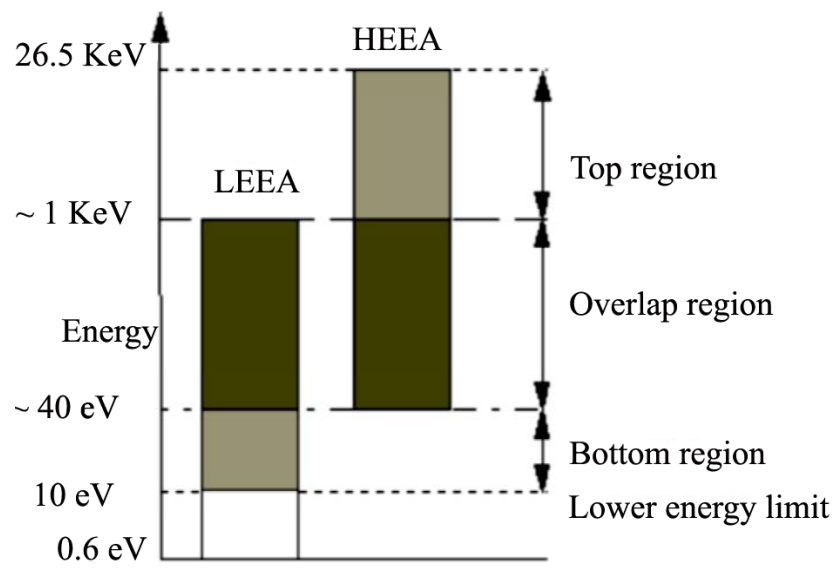

Fig. 2. An example of the energy range coverage for the combination of the two analysers. The moment calculations are calculated from electrons observed with energies of $>10 \mathrm{eV}$, with the bottom region at $10 \mathrm{eV}-40 \mathrm{eV}$, overlap at $40 \mathrm{eV}-1 \mathrm{keV}$ and the top region at $>1 \mathrm{keV}$

subset of the energy range. Onboard moment calculations are made for energies $>10 \mathrm{eV}$, with the subsequent energy range divided into 3 regions depending on energy, as shown in Fig. 2. Due to the sensor mounting geometry, the Top and Bottom energy ranges have a $4 \mathrm{~s}$ resolution (measured only by HEEA and LEEA, respectively) while the Overlap energy range (measured by both sensors) has a $2 \mathrm{~s}$ resolution. We note that data presented in this paper are derived using preliminary calibrations. (For further instrument information, the reader is referred to Johnston et al., 1997; Owen et al., 2001, this issue).

\section{Observations}

\subsection{CASE 1: 26 January 2001}

In this case, we examine Cluster data for the period 08:00 to 11:00 UT. In Fig. 3, we show the IMF conditions (magnetic field magnitude and $3 \mathrm{GSE}$ coordinates) detected at $\mathrm{ACE}$ corresponding to this time period. ACE was $\sim 243 R_{E}$ upstream of the Earth where the solar wind speed was observed to be $\sim 350 \mathrm{~km} / \mathrm{s}$. A lag time to Cluster can be calculated using the solar wind speed and distance from ACE to Cluster $\left(243 R_{E} / 350 \mathrm{~km} / \mathrm{s}=74 \mathrm{~min}\right)$. With the $74 \mathrm{~min}$ lag time, we have indicated in Fig. 3 the corresponding positions of events $\mathrm{A}$ and $\mathrm{F}$ in the Cluster data as shown in Fig. 4. The IMF remains weakly eastward (positive $B_{Y}$ ), along with a transition from weakly positive to weakly negative $B_{Z}$ occuring at $\sim$ 07:37 UT (corresponding to $\sim 08: 51 \mathrm{UT}$ at Cluster), and turning strongly negative at $\sim$ 07:48 UT $(\sim 09: 02$ UT at Cluster). The data from Cluster 3 (Samba) during the 3 hour period from 08:00 to 11:00 UT on 26 January 2001 are shown in Fig. 4. In the upper panel, the black trace shows density moments from the Top region of the energy range $(>1 \mathrm{keV})$ and the red trace shows the Overlap region $(35 \mathrm{eV}-$ 


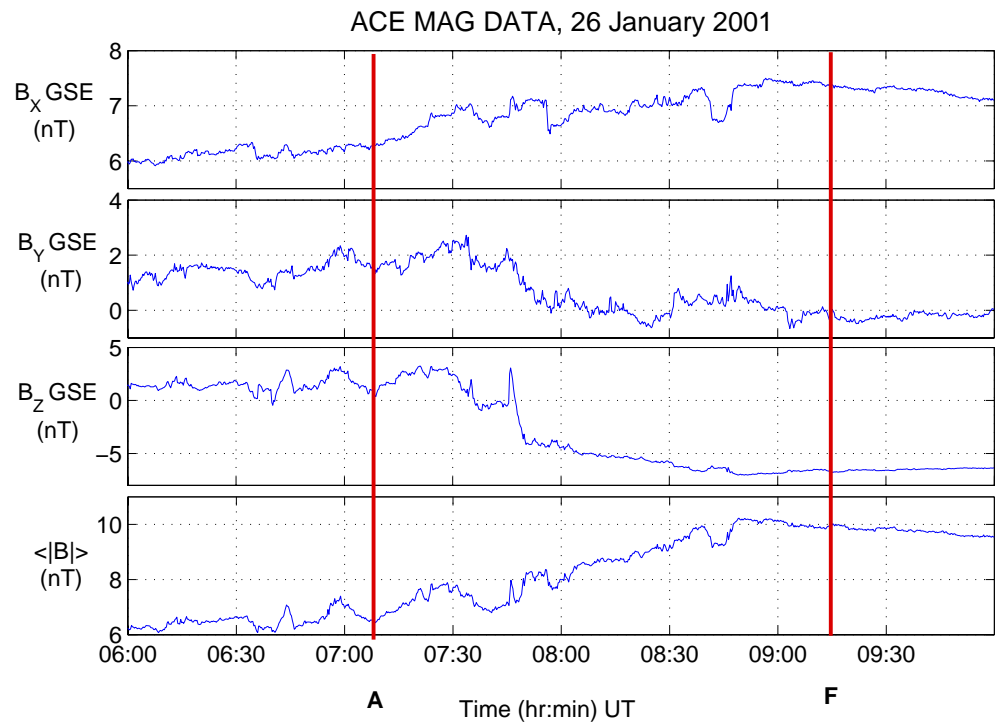

Fig. 3. The IMF conditions detected by the ACE spacecraft for 26 January 2001. Using a $\sim 74$ min lag time calculated from solar wind speed and ACE position, we have marked the region corresponding to the Cluster observations shown in Fig. 4 with red vertical lines.
$1 \mathrm{keV})$. These two ranges give a good indication of the different ambient plasma populations, with typical magnetospheric electron energies $(\sim \mathrm{keV})$ covered by the Top region and the lower energy, magnetosheath electrons ( 10's-100's eV) covered by the Overlap region. The lower panel shows an energy spectra generated from a combination of both HEEA and LEEA sensors from the zone 11 anode, which records electrons travelling in the $+Z$ GSE direction. These energy vs time spectra are presented in a differential number flux $\left(\# / \mathrm{cm}^{2} s\right.$ streV) as indicated by the colour bar on the right, with energy given in electron volts. We note the very high fluxes below $10 \mathrm{eV}$ are due to spacecraft secondary- and photo-electrons. The position of the spacecraft at the bottom of the figure is shown in GSE coordinates.

At the start of the interval shown in Fig. 4, the spacecraft are located in the plasma mantle, and observe characteristically low plasma densities with energies up to about $100 \mathrm{eV}$. At 08:20 UT, marked A in the figure, the spacecraft observes an increase in electron density along with spectral properties showing an injection of magnetosheath-like plasma, with energies up to $200 \mathrm{eV}$. The spacecraft position at this time was $77.3^{\circ}$ invariant latitude and 14.6 MLT. For the upstream IMF conditions described in Fig. 3 (positive $B_{Y}$ and $B_{Z}$ ), these positions place Cluster within the exterior cusp region described by Fung et al. (1997), (12.8 MLT \pm 2.1 hours, $81^{\circ}$ ). The plasma characteristics described above are also consistent with observations of the cusp region (Newell and Meng, 1992). In Fig. 4 at 08:26 UT, Cluster observes a reduction in density from "cusp" values (but greater than lobe values) and also a reduction in peak spectral flux by a factor $\mathrm{O}(100)$. For this preliminary study, we interpret this behaviour as a result of Cluster briefly skimming the duskside flank of the exterior cusp (Fung et al., 1997) and entering the low latitude boundary layer (LLBL) (Newell and Meng, 1992). Between 08:40 UT (marked B) and 09:12 UT (marked C), Cluster observes of a series of brief transitions lasting $\sim$ min into a re- gion with much higher energy electrons (up to $10 \mathrm{keV}$ ), associated with a drop-out in the lower energy, magnetosheathlike electrons $(<200 \mathrm{eV})$. These observations are consistent with the boundary plasma sheet (BPS, e.g. Newell and Meng, 1992). Therefore, we infer that the spacecraft make multiple transient encounters with the boundary between the two plasma populations, signified by the bursty nature of the spectra in which the energetic magnetospheric electrons trapped on closed field lines alternate with a less energetic magnetosheath electron population on open field lines. We note that these events are the opposite of the encounters discussed by Lockwood et al. (2001, this issue) where the Cluster spacecraft are predominantly in the BPS, making brief excursions into the LLBL. Between 09:12 (marked C) and 09:20 UT (marked D), Cluster observes dense, lower energy electrons, indicated by the increase in the Overlap density, accompanied by a reduction in the photoelectron population, suggesting an entrance into the magnetosheath (when compared to the population observed in the magnetosheath proper after 10:30 UT). From 09:20 UT (D) until 09:54 UT (marked E), a further series of transient encounters with BPSlike plasma occurs. Between these encounters, the density is much more magnetosheath-like, when compared to the observations between B and C. This suggests transient encounters between the BPS and the magnetosheath. From 09:54 UT (E) until about 10:30 UT (marked F), Cluster observes variations similar to those observed between $B$ and $C$. Just before 10:30 UT (marked F), observations of a strong density enhancement of lower energy electrons signifies the entrance into the magnetosheath proper.

Figure 5 shows the magnitude and three GSE coordinates of the magnetic field from the Flux Gate Magnetometer (FGM) instrument (Balogh et al., 1997 and Balogh et al., 2001, this issue) on board Cluster 3 (Samba). Overall, observations of the magnetic field are consistent with Cluster being located poleward of the cusp and on its dusk side. Note 


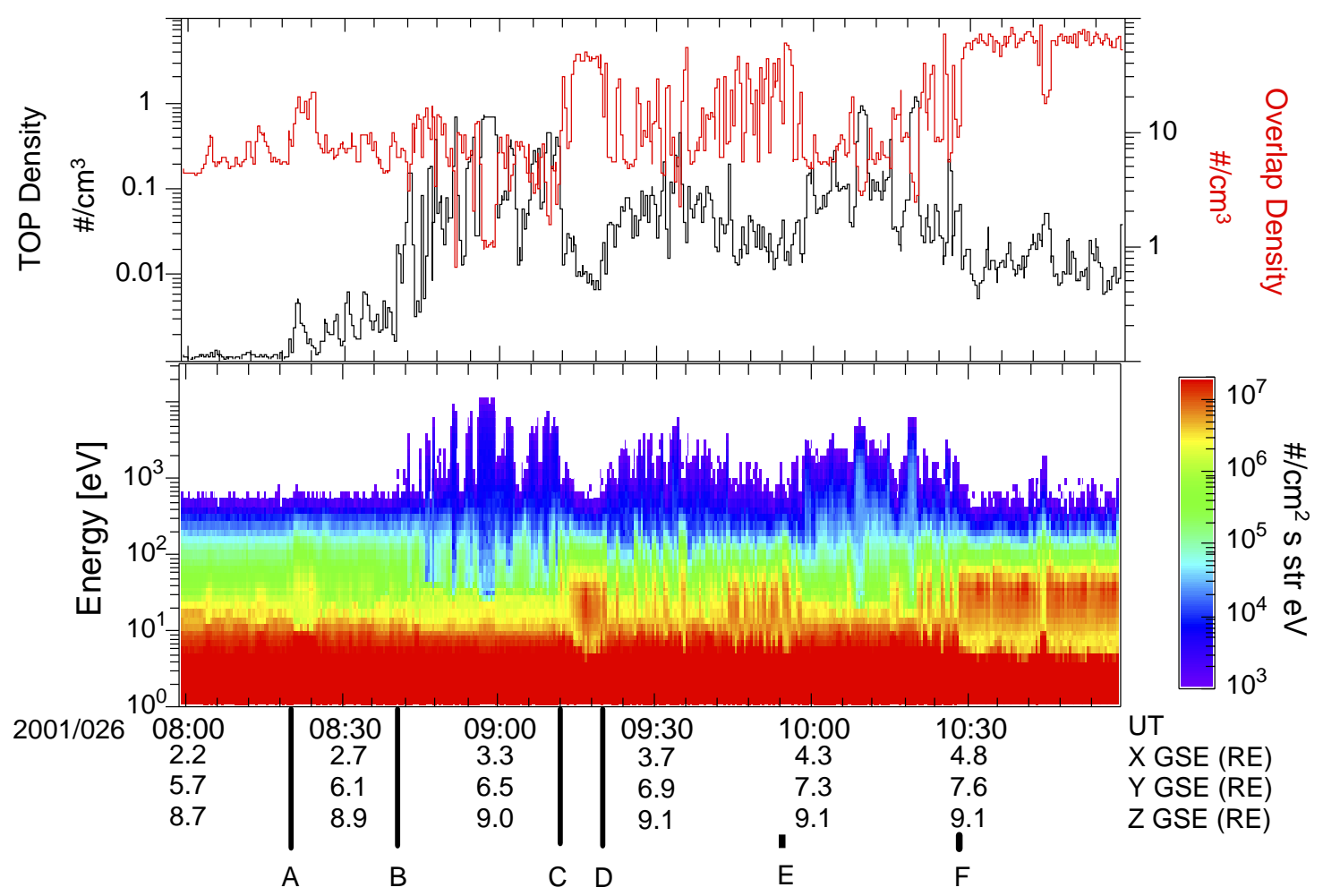

Fig. 4. The density moments and energy spectra from 26 January 2001, 08:01-11:50 UT. The upper panel shows density from the Top energy region in black and the Overlap energy range in red. The spectrogram is a combination of the HEEA and LEEA sensor, measured in a differential number flux $\left(\# / \mathrm{cm}^{2} \mathrm{~s}\right.$ str eV). We note the large band of photoelectrons below $\sim 10 \mathrm{eV}$.

the large deviations in $\mathrm{B}_{X}$ and $\mathrm{B}_{Z}$ just after 09:10 UT correspond to the magnetosheath entry between $C$ and $D$ in Fig. 4 . This is followed by a region of rapid magnetic fluctuations that end around 10:00 UT, coinciding with the appearance of the lower energy population recorded by PEACE, marked $\mathrm{E}$ in Fig. 4. The region of less rapid magnetic fluctuations between 10:00 UT and 10:30 UT corresponds to the interval $\mathrm{E}$ to $\mathrm{F}$ in Fig. 4. The rotation in $B_{X}$ and $B_{Z}$ just after 10:30 UT corresponds to the entry of the magnetosheath proper observed in the electron data. We note the significant magnetic field structure after 10:30 UT and the lack of features in the corresponding electron data. An analysis of these features along with the overall crossing will be presented elsewhere. We now focus on the period just after the entry into the boundary layer at the time marked B in Fig. 4. In Fig. 6, we again show combined HEEA and LEEA spectra in a differential number flux $\left(\# / \mathrm{cm}^{2} \mathrm{~s}\right.$ str $\left.\mathrm{eV}\right)$ this time from all 4 spacecraft (Cluster 1 (Rumba) at the top, through to Cluster 4 (Tango) at the bottom) for the $5 \mathrm{~min}$ period, 08:44-08:49 UT. Differences in measurements from each of the 4 spacecraft are immediately apparent. A flux enhancement centred $\sim 08: 46: 45$ UT (labelled B1) is detected only at Cluster 2 and 3, while the feature centred $\sim 08: 48: 00$ UT (labelled B2) is detected at all four spacecraft. Figure 7 covers the same period but in this case, we display only the Top density moments from each spacecraft (Cluster 1 in the top panel through to Cluster 4 in the bottom panel), which give a good indication of the trapped magnetospheric electron population (energy $>1 \mathrm{keV}$ ), as discussed above. We note the additional features at 08:45:00 and 08:48:30 UT seen only at Cluster 3 (with possible correlated features in Cluster 2).

\subsection{CASE 2: 4 February 2001}

In this case, we examine Cluster data for the period of 08:00 to 11:00 UT. The corresponding IMF conditions recorded at ACE are shown in Fig. 8. The solar wind conditions were quiet at this time with a bulk speed of around $(320 \mathrm{~km} / \mathrm{s})$. Using a similar method to case one, we calculate a lag time to Cluster of $\sim 84 \mathrm{~min}$ and have indicated with the vertical red lines the corresponding position of $\mathrm{A}$ and $\mathrm{E}$ in the Cluster data shown in Fig. 9. From this region we can see that during the Cluster passage, the IMF is persistently northward but sees several changes in the direction of $B_{Z}$. We present an overview of the period from 19:30 to 23:00 UT, 4 February 2001 in Fig. 9. This is in the same format as Fig. 4, with the data taken from Cluster 3 (Samba). At $\sim$ 19:30 UT, Cluster is in a region of low energy plasma. Just before 19:57 UT, marked by A, Cluster enters a region of enhanced electron flux, with energies rising to just below $\sim 1 \mathrm{keV}$. The appearance of this plasma, as in Case 1, indicates an entrance into the cusp. In the present case, we make no distinction between the numerous components in the vicinity of the cusp region (entry layer, etc.). Instead, for this preliminary study, 

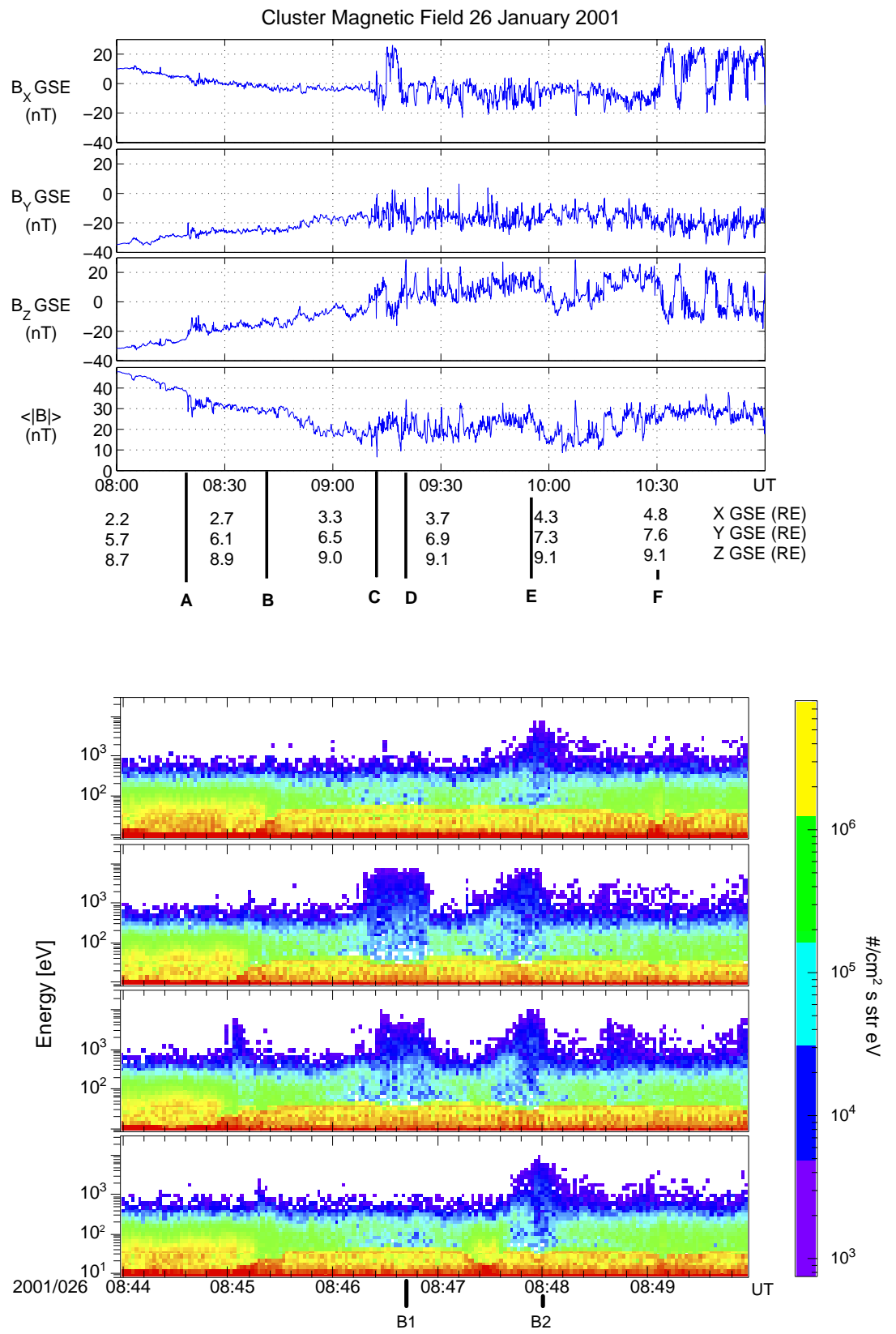

Fig. 5. Magnetic field data from the Flux Gate Magnetometer (FGM). The plot notation is the same for Fig. 3. The $B_{X}$ and $B_{Z}$ components show large deviations just after 09:10 corresponding to the low energy plasma enhancement at "C" in Fig. 4.
Fig. 6. Spectra from all four spacecraft from 26 January 2001, 08:4408:49 UT, focusing on the region B in Fig. 3. Again, the spectra are a combination of the HEEA and LEEA sensors, with Cluster 1 at the top and Cluster 4 at the bottom. The feature at $\sim 08: 46: 41$ (labelled B1) is detected only at Cluster 2 and 3, followed by the event at $\sim$ 08:48:01 (labelled B2) detected at all four spacecraft. we generalise the definition of the cusp given by Fung et al. (1997) and Eastman et al. (2000). We take the cusp to be a region of relatively depressed magnetic field magnitude (Farrell and Van Allen, 1990), with electron energies approaching $1 \mathrm{keV}$ consistent with a mixture of magnetosheath and magnetospheric electrons (Eastman et al., 2000). Around 21:41 UT, marked B, Cluster observes an isolated burst of energetic electrons, which registers a significant increase in the Top density and a large drop-out in the Overlap density. Just before 22:02 UT, marked C, there is a significant decrease in the Top density followed by two transient increases (up to 22:15 UT, marked D), suggesting short duration entries into the magnetosheath and then a final increase back to the energies comparable to those observed between A and B.
However, we note that the region $\mathrm{C}-\mathrm{D}$ retains some higher energy electrons, as indicated by the Top density which decreases, but not to the levels detected in the magnetosheath proper after 22:25 UT (E).

As in Case 1, we gain further insight into the position of the spacecraft at this time by using magnetic field data from the FGM sensor, as shown in Fig. 10a. A smooth reduction in the magnitude of the $B_{Z}$ component of the magnetic field with rather constant $B_{X}$ and a depressed magnetic field magnitude, together with the enhanced electron energies observed by PEACE, indicates passage through the throat of the cusp, as indicated in Fig. 10b. This is supported by the position of Cluster, which is around $82^{\circ}$ invariant latitude and 12.8 MLT throughout this period, very close to the nominal 

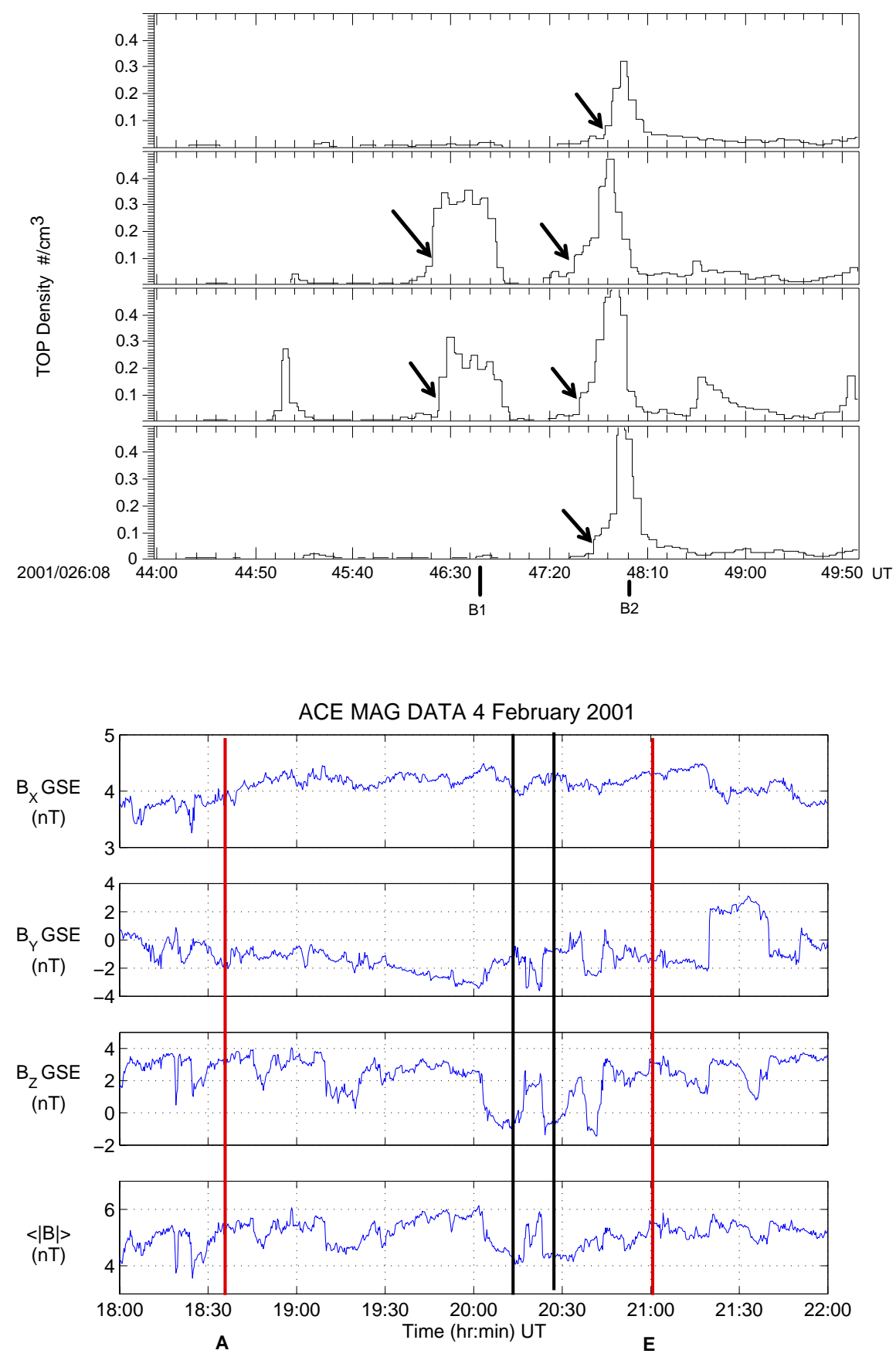

Fig. 7. A plot of the Top density moments for each spacecraft, with Cluster 1 in the top panel, through to Cluster 4 in the bottom panel. The trace represents the densities calculated from electrons detected above $1 \mathrm{keV}$. Timing is carried out such that entry is indicated by a change in the gradient of the ramp seen before the main structure, as indicated by the arrows. A similar method is used for exit. The entry and exit sequence is shown in Table 1.

Fig. 8. IMF conditions from the ACE spacecraft for 4 February 2001, in GSE coordinates. The solar wind speed at this time was $\sim 310 \mathrm{~km} / \mathrm{s}$, giving a lag time to Cluster of $\sim 84 \mathrm{~min}$. Using this time, we have indicated the extent of the Cluster observations in Fig. 9 by the red vertical lines. From this region we can see that during the Cluster passage, the IMF is northward with several changes in the direction of $B_{Y}$. The region bounded by the solid black vertical lines corresponds to $\mathrm{a} \sim 15 \mathrm{~min}$ period around $B$ in Fig. 9, highlighting two $\sim 2$ min variations in the IMF $B_{X}$ and $B_{Y}$. throat of the cusp (Fung et al., 1997). We note the rotation of the magnetic field corresponding to the region of high energy electrons between 21:42 and 21:44 UT (marked B). There are changes in all components of the field, most obviously in $B_{X}$ and a slight increase in the magnitude of the field. Just after 22:00 UT, there is a large rotation in the field corresponding to $\mathrm{C}$ in Fig. 9 followed by a lesser rotation at $\sim 22: 15 \mathrm{UT}$ (D). We note the data gap beginning at 22:30 UT, which occurs during the period covering the magnetosheath entry observed in the electrons, and that the values of the magnetic field data before and after this gap are quite similar. In this case, we focus on the isolated region of high energy electrons at $\sim 21: 45$ UT, marked by B in Fig. 9 and presented in more detail in Figs. 11 and 12. In the latter figures, we show a shorter time interval, from 21:39 to 21:48 UT, with combined HEEA and LEEA spectra from all four spacecraft in Fig. 11 and the Top density moments in Fig. 12. Use of the Top density gives a good indication of the arrival of the high energy electrons at the spacecraft. Viewing the data on this time scale expands the detail of the event itself (between 21:42 and 21:44 UT, marked A) and also reveals a secondary feature between $\sim 21: 44: 15$ and 21:45 UT, marked B. 


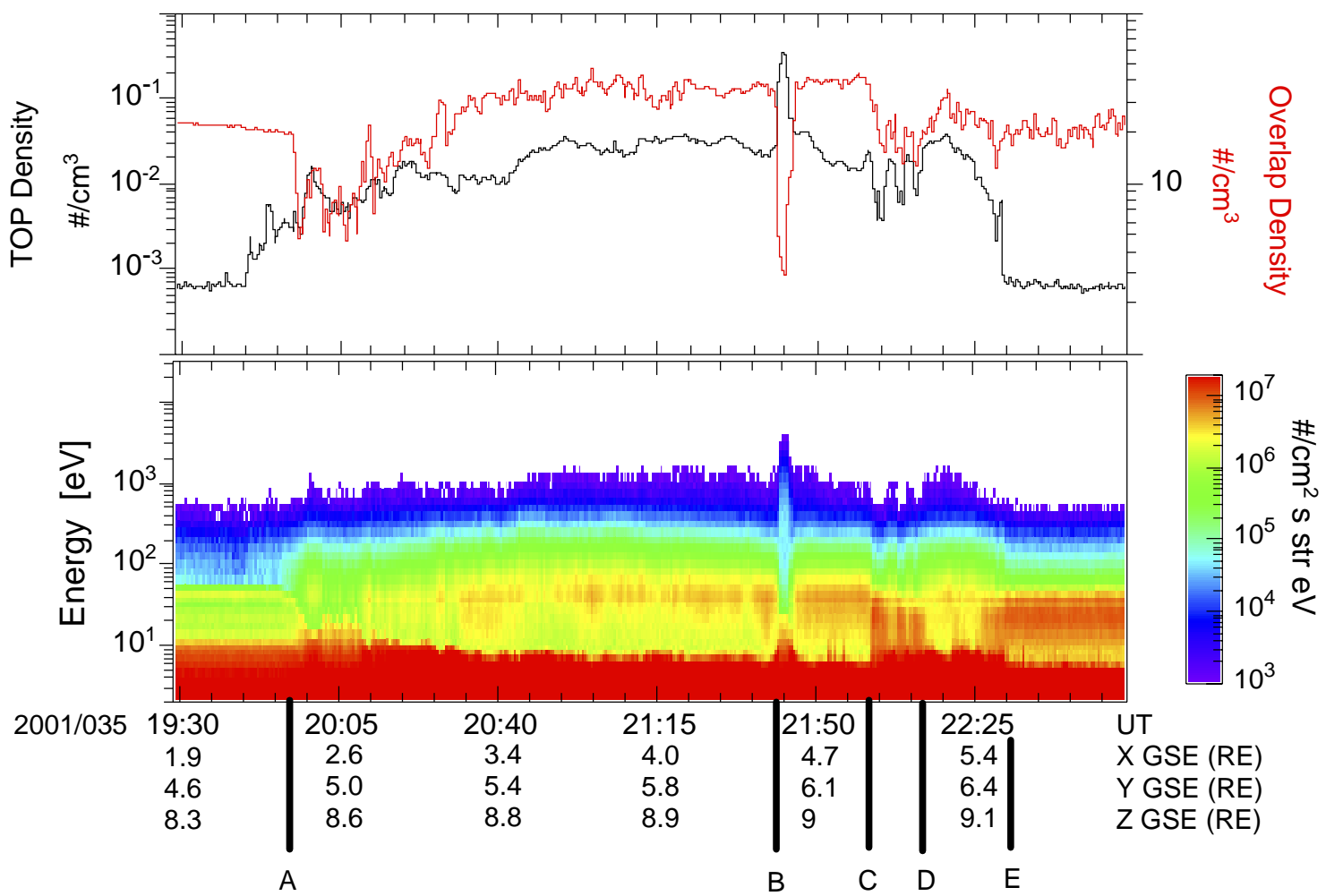

Fig. 9. Energy spectra and density moments from 4 February 2001, 19:30-23:00 UT in same format as Fig. 3.

\section{Discussion}

\subsection{Boundary analysis}

The tetrahedron configuration of the four cluster spacecraft enables the three dimensional study of small scale structures in space plasma for the first time. In this paper we use the method detailed by Harvey (2000), where the spatial gradient of structures encountered by the spacecraft can be determined by the timings and positions of the 4 spacecraft. We assume that the observed boundary is planar such that the boundary normal and velocity are parallel and that they do not change during the passage of the tetrahedron i.e. uniform motion. Following Harvey (2000), we calculate R, a symmetric tensor describing the geometric properties of the tetrahedron

$\mathbf{R}_{j k}=\frac{1}{4} \sum_{\alpha=l}^{4} \mathbf{r}_{\alpha j} \mathbf{r}_{\alpha k}$.

The following equations can then be used to calculate the velocity and direction of the boundary normal.

$m_{l}=\frac{1}{4}\left(\sum_{\alpha=l}^{4} \mathbf{t}_{\alpha} \mathbf{r}_{\alpha k}\right) \mathbf{R}_{k l}^{-1}$,

where $j, k$ and $l$ are matrix indices, $\alpha$ is spacecraft number and

$\mathbf{r}_{\alpha}=\left(r_{\alpha 1}, r_{\alpha 2}, r_{\alpha 3}\right)$
Table 1. Entry and exit sequence of spacecraft for features B1 and B2 in Figs. 6 and 7

\begin{tabular}{c|cccc|cccc}
\hline & \multicolumn{3}{|c|}{ entry sequence } & \multicolumn{4}{c}{ exit sequence } \\
\hline B1 & 2 & 3 & - & - & 2 & 3 & - & - \\
B2 & 2 & 3 & 4 & 1 & 2 & 3 & 4 & 1 \\
\hline
\end{tabular}

where $r \alpha$ and $t \alpha$ are the position and time (with respect to the centre of the tetrahedron) that the $\alpha^{\text {th }}$ spacecraft passes the boundary. Velocity is then given by

$\mathbf{v}=\frac{\mathbf{m}}{|\mathbf{m}|^{2}}$.

In this preliminary analysis, we have neglected detailed consideration of timing and position errors and present the normal direction as a first order approximation of the boundary orientation. This will be improved on in future work.

\subsection{CASE 1: 26 January 2001}

The Top moments are used as an indicator of the appearance of a higher energy electron population from the magnetosphere. The entry-exit timing of each event is inferred by selecting a common feature from each of the structures. For this case, we use the gradient change in the density, as indicated by the arrows in Fig. 7, as a marker for entry and similarly for exit. From these times, we get an entry-exit sequence as indicated in Table 1 . We note that the entry and exit 

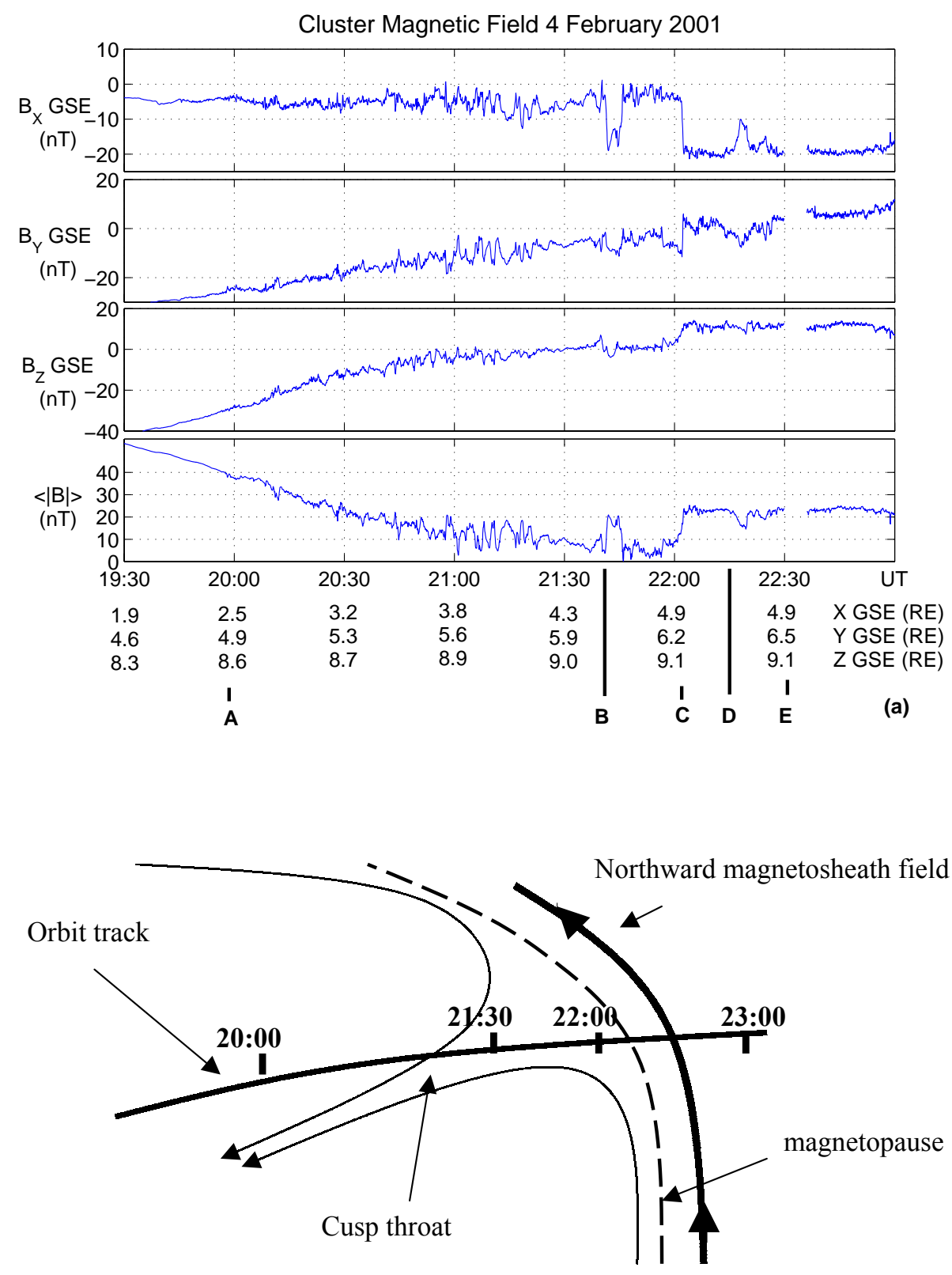

(b)

Fig. 10. (a) Magnetic field data from the FGM sensor on Cluster 3 (Samba). Notation is the same for Fig. 9. We note the change after 21:40, marked B, corresponding to an energetic electron event and the initial entry into the magnetosheath just after 22:00, as inferred from the electron data in Fig. 9. Using this magnetic field data along with the orbit position and MLT data, we propose the orbit path sketched in (b), with the orbit passing directly through the throat of the cusp, with the energetic electron event occurring within this region.

sequence for each spacecraft is identical, i.e. all spacecraft are in the structure for the same length of time (within timings errors, which in this case is 1 satellite spin, $\sim 4 \mathrm{~s}$ ). The direction of motion is calculated to be in the dusk-tailward direction, as indicated in the upper part of Fig. 13. The entry and exit sequence suggests that the boundary is not moving over the configuration in a breathing or back and forth motion. Instead, the differences between B1 and B2 suggest the boundary is wave-like in nature, with varying amplitude. The rapid and bursty nature of these changes in plasma characteristics suggests that Cluster is skimming the boundary, which is travelling duskward and tailward across the satellite orbit trajectory. This is illustrated in the lower diagram in Fig. 13, such that at B1, the Cluster tetrahedron is straddling the boundary and at B2, all the spacecraft enter the magnetosphere. 

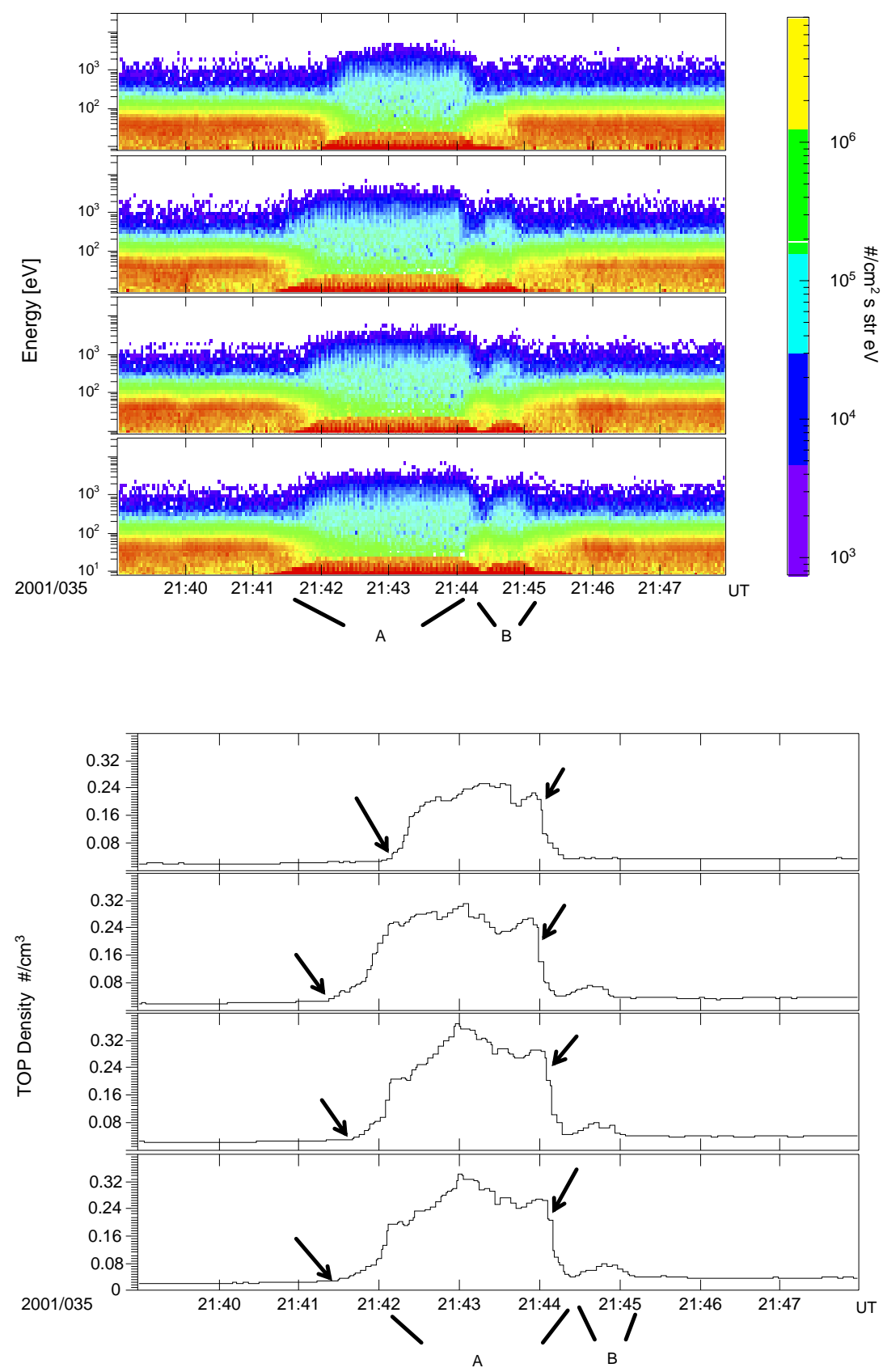

Fig. 11. This figure shows a combination of the HEEA and LEEA sensors for each spacecraft, Cluster 1 at the top through to Cluster 4 on the bottom over time from 21:39 to 21:49 UT We can see the clear differences in both timing and overall structure of the feature in each spacecraft, with the excited electron region between 21:42 and 21:44 UT, marked A. We note the secondary feature between $\sim 21: 44: 15$ and 21:45 (marked B) observable only in spacecraft 2-4.
Fig. 12. The Top region of density (calculated from energies electrons with energies of $>1 \mathrm{keV}$ ), for time between 21:39-21:48 UT, gives a good indication of the extent of the high energy electron population. We use a similar method as was employed for Case 1 to achieve the entry/exit times indicated in Table 2 .
We end the discussion of this case by briefly examining the region observed after $\mathrm{B}$ in Fig. 4, where we observe the appearance of magnetosheath plasma at $\mathrm{C}-\mathrm{D}$ followed by various transitions between BPS and magnetosheath-like plasma (D-E). Similar transients to those observed between B and $\mathrm{C}$ are observed between $\mathrm{E}$ and $\mathrm{F}$ leading up to the magnetopause crossing at F. The $B_{Z}$ component (GSE) of the IMF measured at ACE (Fig. 3) began a large negative turn just before 07:48 UT, with $B_{Y} \sim 0$. Using the lag time calculated above (74 $\mathrm{min}$ ), this $B_{Z}$ shift corresponds to 08:52 UT at Cluster, right in the middle of region B-C in Fig. 4. Due to the basic method of calculating the timing, we cannot infer any direct influence of the IMF conditions on the regions sampled by Cluster at this time. However, it is likely that this IMF change reconfigured the magnetopause boundary, influencing the time at which it was first crossed by Cluster. The bursty appearance of the spectra between D-E suggests that Cluster may be skimming the magnetopause, crossing from the BPS, which is characterised by the high Top density and low Overlap density, to the magnetosheath, signified by the low Top density and a high Overlap density associated with an enhancement of the spectral flux at a few 10 's of $\mathrm{eV}$. We note that during this period, the successive magnetosheath-like signatures show a gradual increase in the Overlap density, which approach the magnetosheath values just before 10:00 UT (E in Fig. 4). This region is then fol- 

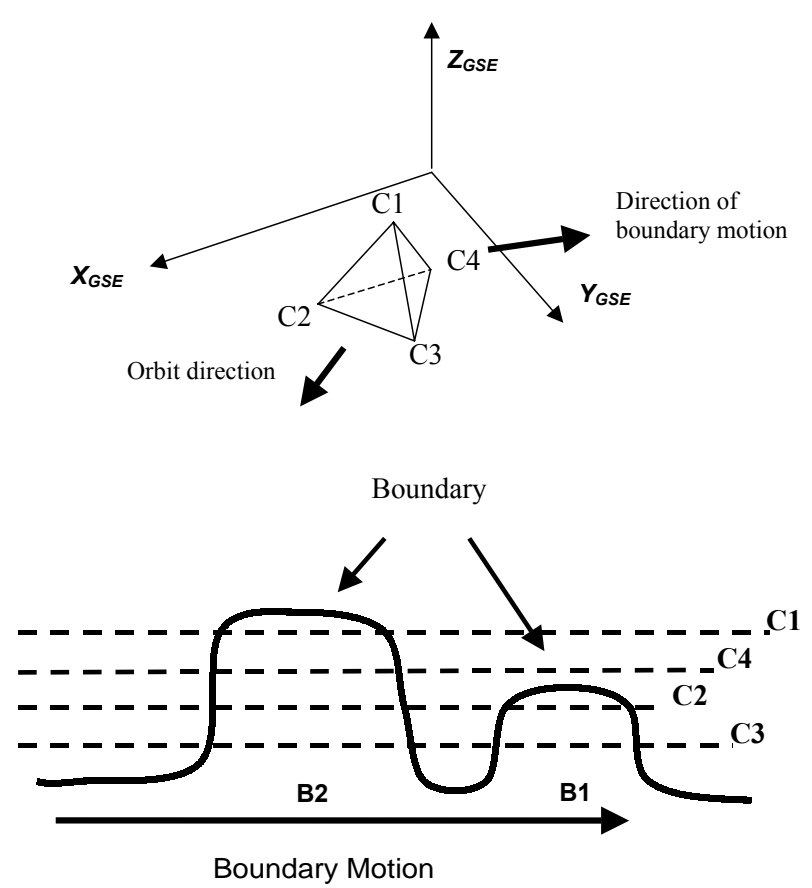

Fig. 13. Diagram in upper panel shows Cluster configuration with the orbit direction, boundary structure, and dusk-tailward motion inferred from timing analysis. The lower panel illustrates the inferred structure of the boundary interaction with Cluster. We propose that the bursty signature is due to the Cluster orbit skimming the boundary between the LLBL/magnetosheath and the BPS.

Table 2. Entry and exit sequence of spacecraft for features A and B from Figs. 11 and 12

\begin{tabular}{c|cccc|cccc}
\hline & \multicolumn{3}{|c|}{ entry sequence } & \multicolumn{4}{c}{ exit sequence } \\
\hline A & 2 & 4 & 3 & 1 & 2 & 1 & 3 & 4 \\
B & 2 & 3 & 4 & - & 2 & 3 & 4 & - \\
\hline
\end{tabular}

lowed by a return of BPS/LLBL transitions between $\mathrm{E}$ and $\mathrm{F}$, which are again accompanied by a gradual increase in the Overlap density corresponding with the appearance of more magnetosheath-like plasma.

Overall, the electron data describes a noisy and turbulent transit through the dusk side boundary of the cusp. The data from PEACE suggest that Cluster is moving out of the magnetosphere, duskward of the cusp through a complicated succession of boundary encounters. During this period, ACE observed a rotation of the IMF into the negative $B_{Z}$ direction. We suggest that this possibly modified the magnetopause in the vicinity of the Cluster spacecraft, further complicating the nature of the magnetopause crossing, inducing a succession of entry/exits from the BPS and LLBL/magnetosheath.

\subsection{CASE 2: 4 February 2001}

From Figs. 11 and 12, the differences in timing and structure between the four spacecraft measurements are evident.

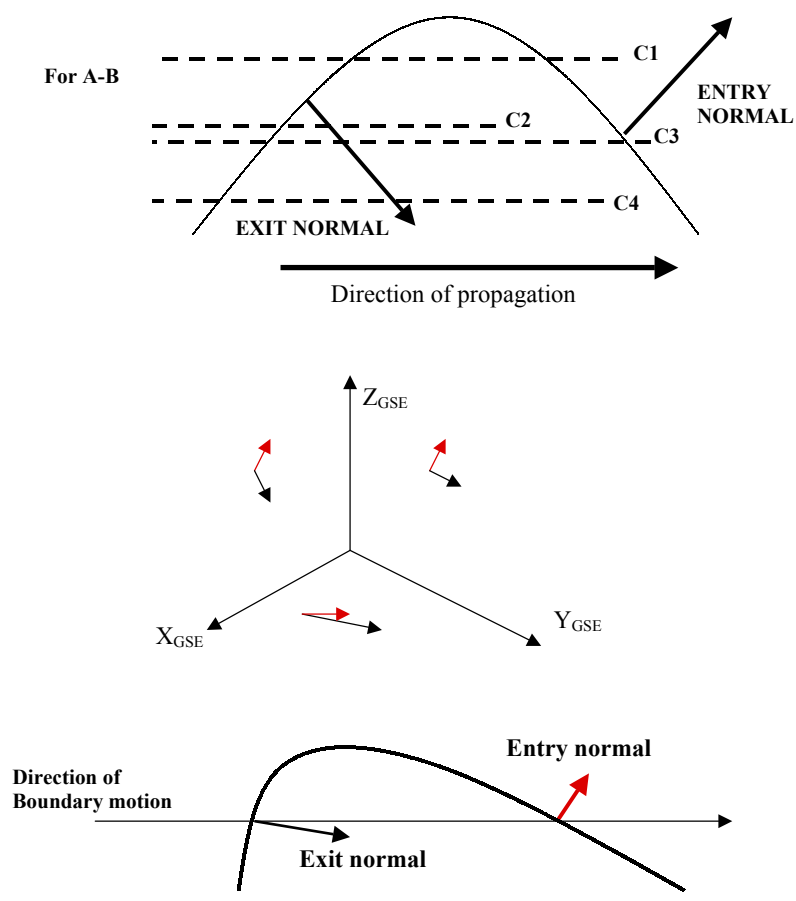

Fig. 14. (a) Illustration of the boundary normal directions for entry and exit of the structure by Cluster, inferring that a wave-or-bulge like feature is passing over the spacecraft. (b) Projection in the GSE plane $(X-Y, Z-Y, Z-X)$ of the boundary normal directions of the entry (red) and exit (black) of event A. We note that the arrows also indicate the velocity magnitude, such that the entry velocity (red) $\sim 10 \mathrm{~km} / \mathrm{s}$, was smaller than exit (black) $\sim 40 \mathrm{~km} / \mathrm{s}$. (c) This difference in velocities can be described by the waveform shown here, with a shallow leading edge and steep trailing edge.

For example, the feature marked at B is not present in spacecraft 1 . We use the above analysis technique to determine the entry and exit sequence using the Top densities shown in Fig. 12. Unlike the previous case, we find that the entry and exit sequences, shown in Table 2, are not the same, indicating that the exit boundary normal of the structure is orientated differently to that of the entry. The rotation of the boundary normal from entry to exit can be explained in terms of a wave or quasi-cylindrical structure passing over the spacecraft, as shown in Fig. 14a. The motion of this structure is directed duskward towards the tail, as indicated in Fig. 14b, where we show the projection of the entry and exit directions of feature A in each GSE plane $(X-Y, Z-Y, Z-X)$, with entry in red and exit in black. The duskward direction of motion can clearly be seen. The size of the arrows indicate the magnitude of the velocity, where the entry velocity was found to be $\sim 10 \mathrm{~km} / \mathrm{s}$ and the exit velocity $\sim 40 \mathrm{~km} / \mathrm{s}$. This suggests the feature to have a geometry shown in Fig. 14c, with a shallow leading edge and a steeper tail.

The energetic nature of the particle populations within this structure compared to the characteristic energies of the surrounding plasma and the reduction in Overlap density, suggest that Cluster has moved from the cusp into a closed magnetospheric electron population, such as the BPS described 
in Case 1. Indeed, one may compare the event at 21:45 UT (B) in Fig. 9 to the event just before 09:00 UT in Fig. 4, with an increase in the Top densities and a decrease in the Overlap densities common to both. However, the present case has a well-defined structure, a much longer duration and perhaps most striking, appears to be convecting along the edge of the cusp.

Using the lag time of 84 min calculated above, the appearance of the BPS corresponds to $\sim 20: 18$ UT at ACE, during which time ACE observed a number of changes in IMF $B_{Y}$ and $\mathrm{B}_{Z}$ as shown in Fig. 8. In particular, we note the modulations in $B_{Y}$ and $B_{Z}$ at $\sim 20: 16$ and $\sim 20: 22$ UT with a duration of $\sim$ min, as indicated by the region between the black vertical lines in Fig. 8. Cluster observations imply motion of the cusp throat, most probably due to IMF variations. The dependence of the cusp location on IMF conditions has been discussed previously by many authors (e.g. Fung et al., 1997; Newell et al., 1989; Zhou et al., 2000). These studies found that for a northward IMF $B_{Y}<0\left(B_{Y}>0\right)$, the cusp shifts dawnward (duskward) in addition to a minor deviation equatorward for decreasing positive $B_{Z}$. However, from our approximate lag timings, it is inappropriate to precisely correlate motion of the exterior cusp with respect to changes in the IMF on these time scales.

Using a combination of the position of the spacecraft (in the throat of the cusp) and the duskward convection of the BPS/magnetospheric plasma structure calculated above, we suggest that Cluster is in the vicinity of the "shoulder" of the magnetosphere bordering the cusp, as shown in Fig. 15a. The observed transient entry of the BPS/magnetosphere is a result of Cluster observing the small-scale structure of the cusp boundary, as shown in Fig. 15b. An obvious candidate for causing this motion is a change in the IMF. A detailed study with accurate timings is required to validate/refute the correlation of these events, but is beyond the scope of this preliminary case study.

\section{Conclusion}

This work has highlighted two events from the northern dusk side, high-latitude magnetosphere/magnetosheath boundary. These events rely on preliminary calibrations of electron data from the PEACE instrument on board Cluster.

In the first case from 26 January 2001, we have presented data from a time where Cluster is flying through alternating BPS and LLBL/magnetosheath plasmas. The traversal of these regions is characterised in the electron data by successive observations of magnetospheric plasma, with a higher energy and lower density, followed by LLBL/magnetosheath plasma, with lower energy and higher density. The data from the magnetometer suggest that Cluster is duskward and slightly poleward of the cusp. In this work, we have focused on a region where initially the Cluster spacecraft are straddling a boundary between the 2 populations (event B1 in Figs. 6 and 7), such that at one point, only 2 of the spacecraft cross over the boundary. Numerous crossings follow this
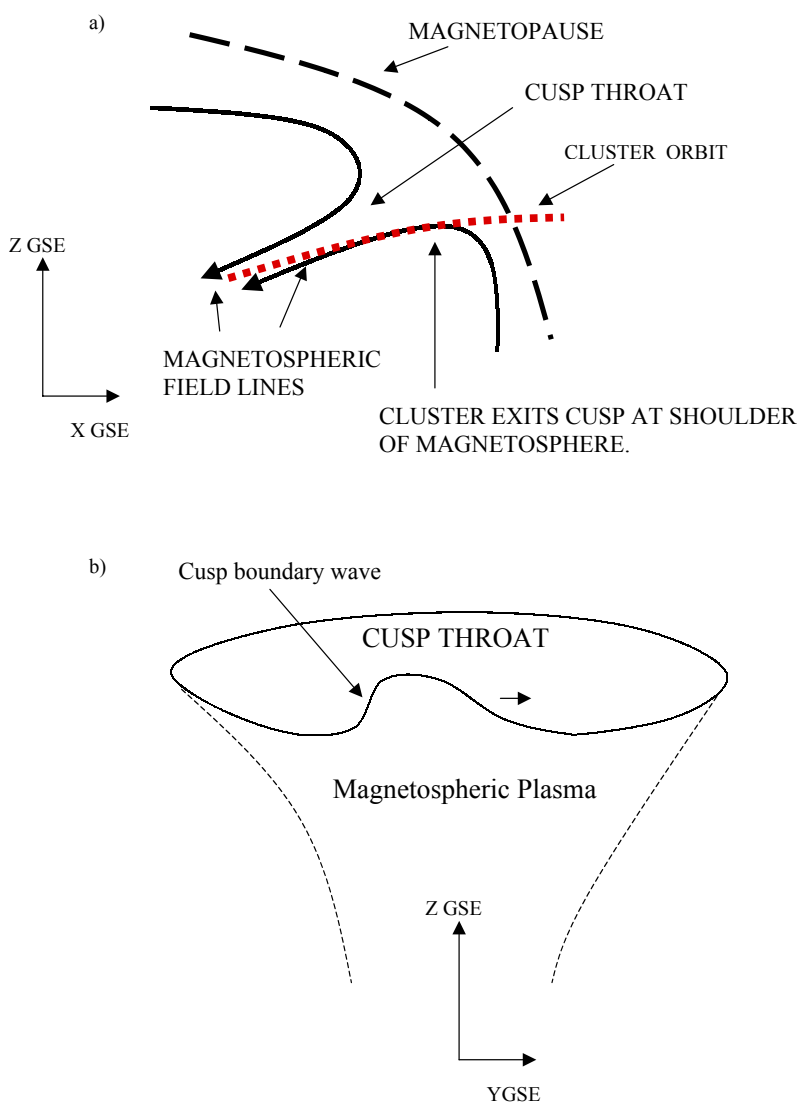

Fig. 15. (a) The diagram shows the cusp viewed from dawn in the $X-Z$ plane, with the solar wind flowing from the right. The appearance of BPS plasma in the cusp suggests an entry into the magnetosphere, as indicated. However, the different orientation of the entry and exit by Cluster to/from the BPS suggests that the transient entry into the BPS is due to a wave-like structure travelling along the equatorward edge of the cusp as in (b). Here, we suggest that the rotation of the IMF modifies the equatorward cusp boundary layer, such that Cluster observes a magnetospheric population convecting duskward along the equatorward edge of the cusp.

event, with the entire tetrahedron traversing the boundary on several occasions. The nature and rapidity of these crossings suggest that Cluster is skimming very close to the boundary. Assuming the boundary to be planar, we find the ordering of the entry and exit sequence of the magnetospheric populations to be identical, suggesting the same boundary normal and direction of motion. This discounts the possibility of the boundary "breathing" back and forth over the spacecraft, and points more towards a boundary wave with very steep edges (as indicated in Fig. 13). In addition, we have tentatively linked a change in the IMF direction to a change in the plasma conditions detected at Cluster, suggesting a change in the position of the magnetopause and a resultant change in plasma characteristics, i.e. appearance of magnetosheathlike plasma.

During the second case observed on 4 February 2001, the orbit position as well as data suggests that the Cluster tetrahedron is in the throat of the cusp. The focus in this example 
was on a region of electron data with significantly enhanced energy (similar to the BPS plasma population observed in Case 1), which is unlike any other plasma population detected elsewhere throughout the entire pass. Using the planar boundary assumption, we determined the entry and exit sequence. However, in this case, they are not the same. Instead, the normal direction changes, such that the structure describes a cylindrical or wave-like configuration, travelling duskward and tailward. The IMF orientation is northward in this case, with several changes in the orientation and magnitude of $B_{Y}$ and $B_{Z}$ (GSE), which we associate with the time period under scrutiny by taking a $84 \mathrm{~min}$ time lag. In particular, we note 2 periods around $\sim 20: 18$ UT, where the variations in $B_{Y}$ and $B_{Z}$ occur over a time scale similar to that of the transient event at Cluster. We suggest that the position of Cluster with respect to the equatorward cusp boundary permits the fortuitous observation of a magnetospheric population convecting duskward along the equatorward edge of the cusp. Due to our basic calculation of lag time, we do not attempt to directly correlate precise IMF changes with observations at Cluster. However, given the time scale and appearance of these observations we suggest that the IMF changes may have had some effect.

The analysis and explanation of the two cases presented in this work and many similar examples require much more study to validate the suggested explanations as to their origin, in particular the effect of IMF variation, and will be presented elsewhere. However, we conclude that they are excellent examples of the potential of the Cluster experiment in unlocking the 3-dimensional picture of the magnetospheric system.

Acknowledgements. The authors would like to thank the two referees for their helpful comments and observations during the submission process of this paper. We gratefully acknowledge the work from the engineering and operations teams at UCL/MSSL in building the PEACE experiment as well as the FGM team. We also acknowledge the Bartol Research Institute (BRI) and Norman F. Ness, Los Alamos National Laboratory and D. J. McComas and particularly the help from R. Skoug for use of the level 2 ACE MAG and ACE Solar Wind Experiment data respectively. This work has been supported in the UK by the UCL/MSSL PPARC Rolling grant.

Topical Editor G. Chanteur thanks two referees for their help in evaluating this paper.

\section{References}

Balogh, A., Dunlop, M., et al.: The Cluster Magnetic field experiment, Space Science Reviews 79, 65-91, 1997.

Balogh, A., Carr, C. M., Acuña, M. H., et al.: The Cluster magnetic field investigation: overview of in-flight performance and initial results, Ann. Geophysicae, this issue, 2001.

Chen, S.H., Boardsen, S. A., Fung, S. F., Green, R. L., Kessel, R. L., Tan, L. C., Eastman, T. E., and Craven, J. D.: Exterior and interior polar cusps: Obervations From Hawkeye, J. Geophys. Res., 102, No. A6, 11, 335-347, 1997.

Dunlop, M. W., Cargill, P., Stubbs T., and Woolliams, P.: The high altitude Cusps: HEOS-2, J. Geophys. Res., 105, 27, 509-517, 2000 .
Eastmann, T. E., Boardsen, S. A., Chen, S.-H., and Fung, S. F.: Configuration of high-latitude and high-altitude boundary layers, J. Geophys. Res., 105, A10, 23 221-23 238, 2000.

Farrell, W. M. and Van Allen, J. A.: Observations of the Earth's polar cleft at large radial distances with the Hawkeye 1 satellite, J. Geophys. Res., 95, 20 945, 1990.

Fung, S. F., Eastman, T. E., Boardsen, S. A., and Chen, S.-H.: Highaltitude Cusp positions sampled by the Hawkeye satellite, Phys. Chem. Earth, 22, 7-8, 1997.

Haerendel, G., Paschmann, G., Sckopke, N., and Rosenbauer, H.: The Frontside Boundary layer of the Magnetopause and the Problem of Reconnection, J. Geophys. Res., 83, 1978.

Harvey, C. H.: Spatial gradients and the Volumetric Tensor, in: Analysis Methods for Multi-Spacecraft Data, (Eds) Paschmann, G. and Daly, P., ISSI, ESA publications division, 1998.

Hedgecock, P. C. and Thomas, B. T.: HEOS Observations of the Configuration of the Magnetosphere, Geophys. J. R. Astr. Soc., Vol. 41, 1975.

Johnston, A. D., Alsop C., et al.: PEACE: a Plasma electron and current experiment, Space Science Reviews, 79, 351-398, 1997.

Kessel, R. L., Chen, S.H., Green, J. L., Fung, S. F., Boardsen, S. A., Tan, L. C., Eastman, T. E., Craven, J. D., and Frank, L. A.: Evidence of highaltitude reconnection during northward IMF: Hawkeye observations, 23, 5, 583-586, 1996.

Lockwood, M., Fazakerley, A., Opgenoorth, H., Moen, J., van Eyken, A. P., Bosqued, J.-M., Lu, G., Eglitis, P., McCrea, I. W., Cully, C., Hapgood, M. A., Wild, M. N., Stamper, R., Taylor, M., Wild, J., Provan, G., Amm, O., Kauristie, K., Pulkkinen, T., Strømme, A., Prikryl, P., Pitout, F., Dunlop, M., Balogh, A., Rème, H., Behlke, R., Denig, W., Hansen, T., Greenwald, R., Morley, S. K., Alcaydé, D., Blelly, P.-L., Donovan, E., Engebretson, M., Lester, M., Waterman, J., and Marcucci, M. F.: Coordinated Cluster and ground-based instrument observations of transient changes in the magnetopause boundary layer during northward IMF: relation to reconnection pulses and FTE signatures, Ann. Geophysicae, this issue, 2001.

Merka, J., Safrankova, J., Nemecek, Z., et al.: High-altitude cusp: INTERBALL observations, Adv. Space Res., 25, No. 7-8, 14251434, 2000.

Newell, P. T., Meng, C.-I., Sibeck, D. G., et al.: Some low-altitude cusp dependencies on the interplanetary magnetic field, J. Geophys. Res., 94, 8921-8927, 1989.

Newell, P. T. and Meng, C.-I.: Mapping the dayside ionosphere to the magnetosphere according to particle precipitation characteristics, Geophys. Res. Lett., 19, 6, 609-612, 1992.

Owen, C. J., Fazakerley, A. N., Carter, P. J., et al.: Cluster PEACE observations of electrons during magnetosphere flux transfer events, Ann. Geophysicae, this issue, 2001.

Russell, C. T.: Polar Eyes the Cusp, Proc. Cluster-II workshop on multiscale / multipoint plasma measurements, London, 22-24 September 1999, ESA SP-449, 47, 2000.

Sandahl, I., Popielawska, B., Budnick, Yu., et al.: The cusp as seen from INTERBALL, Proc. Cluster-II Workshop on Multiscale/Multipoint Plasma Measurements, London, 22-24 September 1999, ESA SP-449, 39-45, 2000.

Zelenyi, L. M., Trska P., and Petrukovich, A. A.: INTERBALL dual probe and dual mission, Adv. Space Res., 20, 4/5, 549-557, 1997.

Zhou, X. W., Russell C. T., and Le, G.: Solar wind control of the polar cusp at high altitude, J. Geophys. Res., 105, 245-251, 2000. 